\title{
Statistical properties of sampled networks by random walks
}

\author{
Sooyeon Yoon, Sungmin Lee, Soon-Hyung Yook $*$ and Yup Kim丹 \\ Department of Physics and Research Institute for Basic Sciences, Kyung Hee University, Seoul 130-701, Korea
}

(Dated: September 7, 2018)

\begin{abstract}
We study the statistical properties of the sampled networks by a random walker. We compare topological properties of the sampled networks such as degree distribution, degree-degree correlation, and clustering coefficient with those of the original networks. From the numerical results, we find that most of topological properties of the sampled networks are almost the same as those of the original networks for $\gamma \lesssim 3$. In contrast, we find that the degree distribution exponent of the sampled networks for $\gamma>3$ somewhat deviates from that of the original networks when the ratio of the sampled network size to the original network size becomes smaller. We also apply the sampling method to various real networks such as collaboration of movie actor, world wide web, and peer-topeer networks. All topological properties of the sampled networks show the essentially same as the original real networks.
\end{abstract}

PACS numbers: 05.40.Fb,89.75.Hc,89.75.Fb

Since the concept of complex network [1] came into the limelight, many physically meaningful analyses for the complex networks in real world have emerged. The examples of such studies include the protein-protein interaction networks (PIN) 2], world wide web (WWW) 3], email network [4], etc. The empirical data or the information of the real networks can be collected in various ways, for example, the traceroutes for the Internet [5] and high throughput experiments for protein interaction map [6]. Thus, it is natural assumption that the empirical data can be incomplete due to various reasons which include some limitations of the experiments and experimental errors or biases. As a result, many real networks which have been intensively studied so far can be regarded as sampled networks. Moreover, several studies have shown that the dynamical properties on the networks can be significantly affected by the underlying topology [7, 8]. Therefore, it is very important and interesting to study the topological differences between the sampled networks and the whole networks.

Recently, several sampling methods such as random node sampling [9, 10], random link sampling, and snowball sampling were studied [10]. The random node sampling is the simplest method in which the sampled network consists of randomly selected nodes with a given probability $p$ and the original links between the selected nodes. On the other hands, in the random link sampling, the links are randomly selected and the nodes connected to the selected links are kept. These two random sampling methods have been used to study the statistical survey in some social networks. In the random sampling method, however, many important nodes such as hubs cannot be sampled due to the even selection probability. Some recent studies show that some networks such as PIN, the topological properties of randomly sampled net-

\footnotetext{
*Electronic address: syook@khu.ac.kr
}

${ }^{\dagger}$ Electronic address: ykim@khu.ac.kr works significantly deviate from those of the original networks [9, 10]. The idea of the snowball sampling method 10, 11] is similar to the breath-first search algorithm [12, 13]. In the snowball sampling method all the nodes directly connected to the randomly chosen starting node are selected. Then all the nodes linked to those selected nodes in the last step are selected hierarchically. This process continues until the sampled network has the desired number of nodes 10. Previous studies showed that the topological properties of the sampled networks closely depend on the sampling methods [10].

In this paper, we focus on the effect of the weighted sampling on the topological properties of sampled networks. In order to assign nontrivial weight to each node, we first note the structure of the real networks. Many real networks are known to be the scale-free networks in which the degree distribution follows the power-law [1],

$$
P(k) \sim k^{-\gamma} .
$$

Moreover, the probability $p_{v}(k)$ that a random walker (RW) visits a node of degree $k$ [7] is given by

$$
p_{v}(k) \sim k .
$$

The degree $k$ causes uneven probability of finding a node by a RW on the heterogeneous networks. Thus, by using the RW for sampling we can assign automatically the nontrivial weight to each node which is proportional to the degree of the node. Due to the uneven visiting probability, the nodes having the large degree, i.e., topologically important parts, can be easily found regardless of the starting position of the RW. Therefore, we expect that the sampling by the RW can provide more effective way to obtain the sub-networks which have almost the same statistical properties as the original one. Furthermore, we also study the effects of the heterogeneity of the original networks on the RW sampling method (RWSM) by changing $\gamma$. This weighted sampling method is also shown to be successfully applied to obtain the important informations of many real networks such as actor net- 
works, WWW, and peer-to-peer (P2P) networks. Therefore, we expect that this study can provide a better insight to understand important properties of the real networks and offer a systematic approach to the sampling of networks with various $\gamma$.

We now introduce the RWSM. First, we generate orignal scale-free networks (SFN) by use of the static model in Ref. 14 from which various sizes of sub-networks are sampled. The size or number $N_{o}$ of nodes of the original network with each $\gamma$ is set to be $N_{o}=10^{6}$. The typical values of $\gamma$ used in the simulations are $\gamma=2.23,2.51,3.05,3.40$, and 4.2 . We set the average degree $\langle k\rangle=4$ for each network. After the preparation of original networks, a RW is placed at a randomly chosen node and moves until it visits $N_{s}$ distinct nodes. Then we construct sub-networks with these $N_{s}$ visited nodes and the links which connect any pair of nodes among the $N_{s}$ visited nodes in the original network. We use $N_{s}=10^{3}, 10^{4}, 2 \times 10^{4}, 4 \times 10^{4}, 6 \times 10^{4}, 8 \times 10^{4}, 10^{5}$, and $1,2,3, \cdots, 9 \times 10^{5}$.

The degree distribution is one of the most important measure for the heterogeneity of networks [1]. In Fig. 1. we compare the degree distributions of the sampled networks to those of the original networks for various $\gamma$. We find that the degree distribution of the sampled network also satisfies the power-law, $P(k) \sim k^{-\gamma_{s}}$.

Especially, from the data in Figs. 1 (a)-(d) we find that the $\gamma_{s}$ of the sampled networks with $N_{s} / N_{o} \geq 0.01$ is nearly equal to $\gamma$ of the original network, even though the $\gamma_{s}$ for the small $N_{s}$ has relatively larger error bar. It shows that the sampling method by RW does not change the heterogeneity in degree for networks with $2<\gamma \lesssim 3$. Since most of the real networks have $2<\gamma<3$ [1], this result is practically important. We summarize the obtained $\gamma_{s}$ 's for various $N_{s}$ 's and $\gamma$ 's in Table 【.

In contrast to the case $\gamma \lesssim 3, \gamma_{s}$ for $\gamma>3$ slightly deviates from the $\gamma$ of the original networks if $N_{s} / N_{o} \lesssim$ 0.1. (See the data for $\gamma=4.2$ in Figs. 1 (e) and (f) or in Table (I) Numerically we find that $\gamma_{s}$ is nearly equal to the original $\gamma$ for $N_{s} / N_{o}>0.1$ when $\gamma \lesssim 4.2$. Of course one can expect the substantial deviation of $\gamma_{s}$ from $\gamma$ as $\gamma$ increases further from $\gamma=4.2$.

This $\gamma$ dependent behavior of $P(k)$ can be understood from Eqs. (11) and (2). Equation (11) indicates that $\left\langle k^{2}\right\rangle$ diverges with finite $\langle k\rangle$ for $\gamma \leq 3$. This implies that the topology of a network has several dominant hubs which have extraordinary large number of degrees when $\gamma<$ 3. Since the probability of visitation of the RW follows Eq. (2), the RW can more effectively find the central part of the network around the hubs when $\gamma<3$. Thus the sampled networks can inherit easily the topological properties of the original networks.

RWSM is also applied to real networks. In Fig. 2 we show the $P(k)$ of the actor network 15], the WWW [3], and the P2P networks (Gnutella) 16]. The number of nodes in the original real networks are $N_{o}=$ 392340, 325729, and 1074843 for the actor network, the WWW, and the Gnutella, respectively. The degree dis-
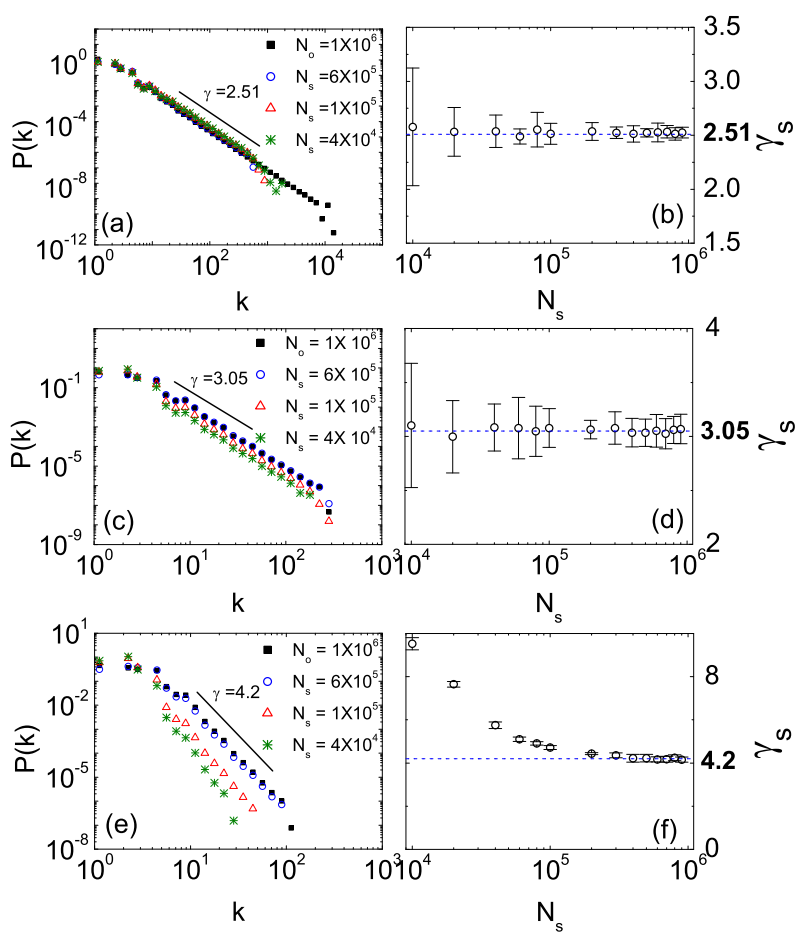

FIG. 1: (Color online) Degree distributions for sampled networks of static scale-free networks with (a) $\gamma=2.51$, (c) 3.05, and (e) 4.2. Degree exponents $\gamma_{s}$ for the sampled networks extracted from the original network for the network size $N_{o}=10^{6}$ with (b) $\gamma=2.51$, (d) $\gamma=3.05$, and (f) $\gamma=4.2$. The slopes of solid lines in (a,c,e) and the values of the dashed lines in $(b, d, f)$ are the degree exponents of the original networks.

tributions for the actor network and the WWW follow the power-law with $\gamma=2.2$ (actor) [15] and $\gamma=2.6$ (WWW) [3]. The data in Fig. 2 (a) shows that $P(k)$ of the sampled actor network follows the power-law with $\gamma_{s}=2.15$ for $N_{s}>10^{3}$. This value of $\gamma_{s}$ is quite close to $\gamma=2.2$. In contrast $\gamma_{s}$ seems to deviate from $\gamma$ of the original network for small $N_{s}\left(=10^{3}\right)$. However, the degree exponent $\gamma_{s}$ for $N_{s}=10^{3}$ still has almost the same value with that of the original network over one decade $(k=10 \sim 100)$. In the case of the WWW, the $\gamma_{s}$ of the sampled networks well agrees with $\gamma \simeq 2.6$ even for small $N_{s}\left(=10^{3}\right)$ (see Fig. 2(b)). For the Gnutella, $P(k)$ of the original network does not follow the simple power-law (11). However, as one can see in Fig. 2 (c), the Gnutella network also has big hubs which cause high heterogeneity in degree, and the sampled networks nearly show the same degree distribution as the original one. These results also provide the evidence that the nodes with large degrees play an important role in the RWSM.

Another important measure to characterize the topo- 

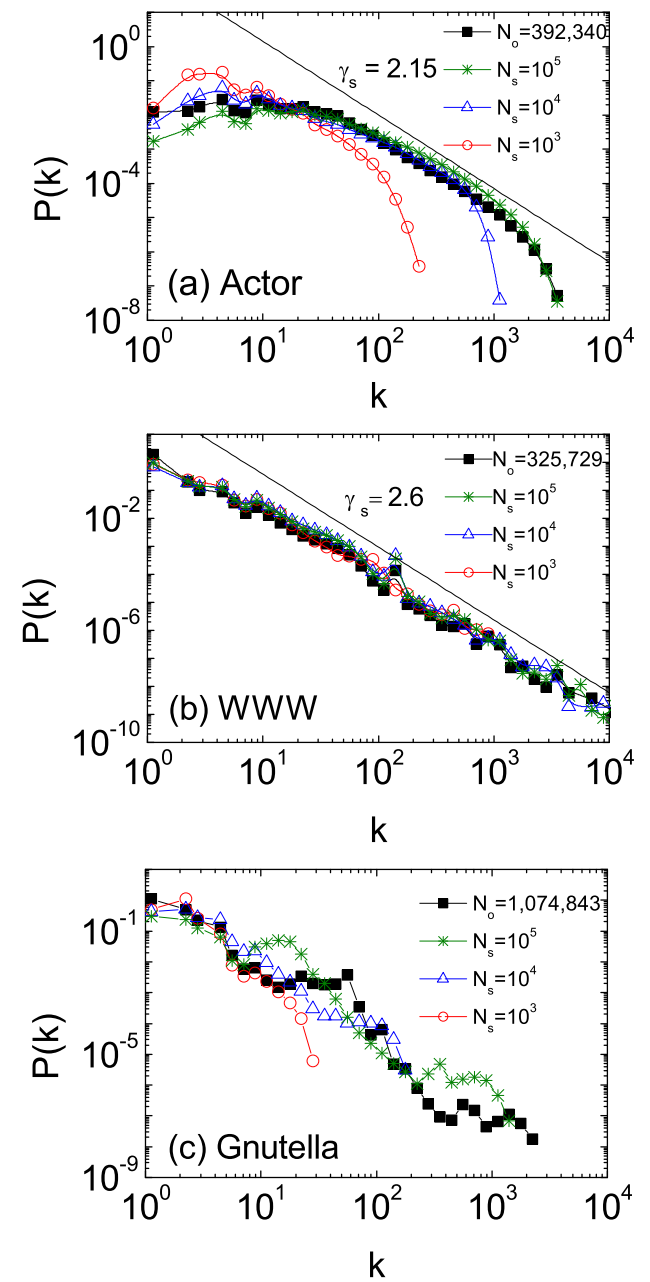

FIG. 2: (Color online) Degree distributions for sampled networks of three real networks. (a) Collaboration network of movie actors $\left(N_{o}=392,340, \gamma=2.2\right)$ 15], (b) WWW $\left(N_{o}=\right.$ $325,729, \gamma=2.6)$ [3], and (c) Gnutella $\left(N_{o}=1,074,843\right)$ [16]. The slopes of the solid lines in (a) and (b) are the values of degree exponents obtained from the simple linear fitting for degree distributions of the sampled networks.

logical properties of the complex network is the degreedegree correlation. Many interesting topological properties such as the self-similarity [17] can be affected by the degree-degree correlation. The degree-degree correlation can be characterized by $\left\langle k_{n n}(k)\right\rangle$, the average degree of the nearest-neighbors of nodes with degree $k$ [18, 19]. If the $\left\langle k_{n n}(k)\right\rangle$ increases (decreases), the network is characterized as assortative (disassortative) mixing. As shown in Fig. 3 (a), for the static SFN with $2<\gamma<3$ the original network and the sampled networks all show the disassortative mixing. This can be explained by the dy- namical properties of RWs on complex networks. In the networks showing disassortative mixing, the RW on a hub should go through a node of small $k$ to move to another hub. Thus, many nodes having small $k$ can be connected to the hubs in the sampled networks and the sampled networks remain disassortative. If the networks have neutral degree correlation, then the networks sampled by the RW also show neutral degree correlation. (See Figs. 33(b) and (c).) In Figs. 3 (d)-(f), we plot $\left\langle k_{n n}(k)\right\rangle$ of real networks. $\left\langle k_{n n}(k)\right\rangle$ 's of the sampled networks show the same degree correlations as those of the original networks. As shown in Figs. 3 (d)-(f), the degree correlations are assortative, disassortative, and neutral for the actor, WWW, and Gnutella networks, respectively.
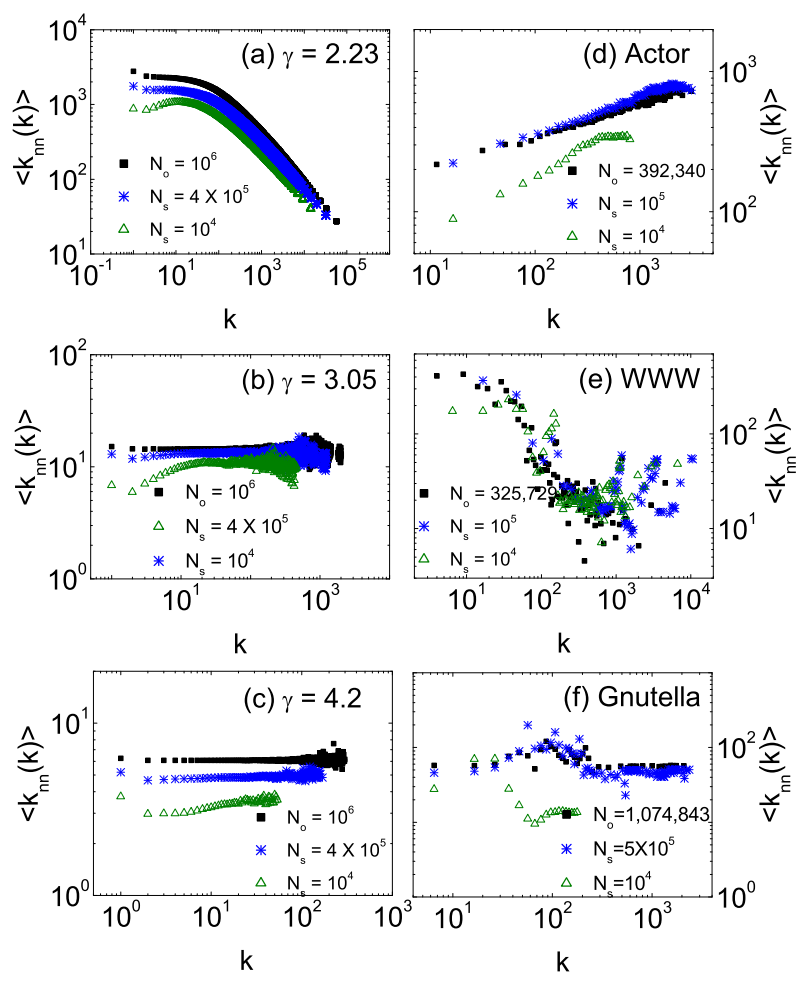

FIG. 3: (Color online) Distributions of $\left\langle k_{n n}\right\rangle$ for sub-networks extracted from the original networks with (a) $\gamma=2.23$, (b) $\gamma=3.05$, and (c) $\gamma=4.2$. (d) Collaboration network of movie actors. (e) WWW. (f) Gnutella.

We also measure a clustering coefficient of the sampled networks. The clustering coefficient $C_{i}$ of a node $i$ is defined by

$$
C_{i}=\frac{2 y_{i}}{k_{i}\left(k_{i}-1\right)},
$$

where $k_{i}$ is the degree of node $i$ and $y_{i}$ is the number of connections between its nearest neighbors [1]. $C_{i}$ physically means the fraction of connected pairs among pairs 
of node $i$ 's neighbors. $C_{i}$ is one if all neighbors are completely connected, whereas $C_{i}$ becomes zero on a infinitesized random network [1].

In Fig. 4, we plot the clustering coefficient $C(k)$ against degree $k . C(k)$ is known to reflect the modular structure of networks [18, 20]. $C(k)$ does not depend on $k$ if the network does not have any well defined hierarchical modules [18, 20]. As shown in Fig. 4. $C(k)$ of both the original networks and the sampled networks shows a tendency to decrease with increasing $k$ for SFN with $\gamma=2.23$ and real networks. (See Figs. 4 (a) and (d)-(f)). This implies that the sampled networks have the same modular structure with original networks. On the other hand, the topology of networks with $\gamma \gg 3$ resembles closely the random graph, thus $C(k)$ does not depend on the degree $k$ [20]. The dependence of $C(k)$ on $k$ for the sampled SFNs with $\gamma \geq 3$ is also nearly the same as the original SFNs. (See Figs. 4 (b) and (c).)
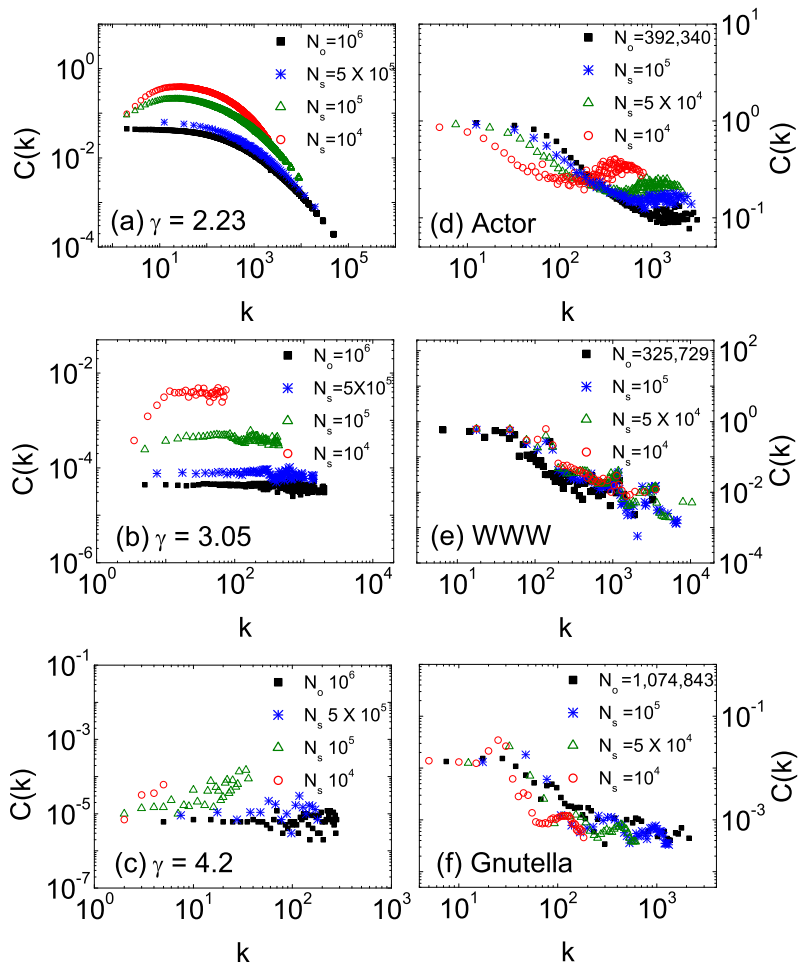

FIG. 4: (Color online) $C(k)$ for sub-networks from the original networks with (a) $\gamma=2.23$, (b) $\gamma=3.05$, and (c) $\gamma=4.2$. (d) Collaboration network of movie actors. (e) WWW. (f) Gnutella.
We study the topological properties of sampled networks by RWSM with SFN and several real networks. From the numerical simulations, we find that the $P(k)$ of the sampled network follows the power-law, $P(k) \sim k^{-\gamma_{s}}$. We also find that the $\gamma_{s} \simeq \gamma$ for all $N_{s}$ when $2<\gamma \lesssim 3$. Even though $\gamma_{s}$ somewhat increases as decreasing $N_{s}$ for $\gamma>3$, the $\gamma_{s}$ 's with $N_{s} / N_{o} \gtrsim 0.1$ still follow the original one. We also study the degree-degree correlation and clustering coefficient by measuring $\left\langle k_{n n}(k)\right\rangle$ and $C(k)$. The sampled networks have the same degree correlation and modular structure with the original networks for all values of $\gamma$. The RWSM is also applied to the actor, WWW, and Gnutella networks. By measuring $P(k)$, $\left\langle k_{n n}(k)\right\rangle$, and $C(k)$, we confirm that the topological properties of the sampled networks are well maintained after sampling and the RWSM is efficient sampling method for the real networks.

The $\gamma$ dependent behavior of the sampled networks can be understood from the dynamical property of a RW. Since most of the networks in the real world have $2<\gamma<3$, the results imply very important meaning in practice. Based on our results, we expect that if we obtain the empirical data by weighted sampling in which the weight is proportional to the degree, then the sampled networks can share the same topological properties with the whole network. Especially, the weighted sampling method becomes very efficient as the heterogeneity of networks increases. At the same time, we also expect that our study can provide a systematic way to extract sub-networks from the empirical data and to study various dynamical properties of the real networks [21].

\section{Acknowledgments}

This work is supported by grant No. R01-2006-00010470-0 from the Basic Research Program of the Korea Science \& Engineering Foundation.
[1] R. Albert and A. -L. Barabási, Rev. Mod. Phys. 74, 47 (2002); S. N. Dorogovtsev and J. F. F. Mendes, Adv.
Phys. 51, 1079 (2002).

[2] S. H. Yook, Z. Oltvai, and A.-L. Barabási, Proteomics 4, 
TABLE I: The changes of the degree distribution exponents $\gamma_{s}$ depending on sampled network size $N_{s}$. $\gamma^{\prime}$ 's are the degree exponents of the original network with $N_{o}=10^{6}$.

\begin{tabular}{c|cccccccccc}
\hline \hline & \multicolumn{10}{|c}{$N_{s}\left(\times 10^{6}\right)$} \\
$\gamma$ & 0.8 & 0.6 & 0.4 & 0.2 & 0.1 & 0.08 & 0.06 & 0.04 & 0.02 & 0.01 \\
\hline $2.23(5)$ & $2.23(3)$ & $2.24(3)$ & $2.24(2)$ & $2.24(3)$ & $2.3(1)$ & $2.2(2)$ & $2.3(2)$ & $2.3(3)$ & $2.3(5)$ & $2.3(5)$ \\
$2.51(7)$ & $2.51(6)$ & $2.53(8)$ & $2.51(8)$ & $2.54(8)$ & $2.5(1)$ & $2.6(2)$ & $2.49(7)$ & $2.5(1)$ & $2.5(1)$ & $2.6(5)$ \\
$3.05(9)$ & $3.1(1)$ & $3.1(2)$ & $3.0(1)$ & $3.06(9)$ & $3.1(2)$ & $3.1(2)$ & $3.1(3)$ & $3.1(2)$ & $3.0(3)$ & $3.1(6)$ \\
$3.40(8)$ & $3.37(7)$ & $3.40(9)$ & $3.4(1)$ & $3.4(1)$ & $3.4(2)$ & $3.5(3)$ & $3.4(2)$ & $3.7(4)$ & $3.8(4)$ & $4.4(3)$ \\
$4.2(1)$ & $4.2(1)$ & $4.2(1)$ & $4.2(2)$ & $4.44(5)$ & $4.71(9)$ & $4.91(8)$ & $5.1(1)$ & $5.8(2)$ & $7.7(1)$ & $9.5(3)$ \\
\hline \hline
\end{tabular}

928 (2003).

[3] R. Albert, H. Jeong, and A.-L. Barabási, Nature 401, 130 (1999).

[4] H. Ebel, L. -I. Mielsch, and S. Bornholdt, Phys. Rev. E 66, 035103(R) (2003).

[5] L. Dall'Asta, I. Alvarez-Hamelin, A. Barrat, A. Vázquez, and A. Vespignani, Phys. Rev. E 71, 036135 (2005); A. Clauset and C. Moore, Phys. Rev. Lett. 94, 018701 (2005).

[6] P. Uetz et al., Nature 403, 623 (2000).

[7] J. D. Noh and H. Rieger, Phys. Rev. Lett. 92, 118701 (2004); J. Lahtinen, J. Kertész, and K. Kaski, Phy. Rev. E 64, 057105 (2001); B. Tadić, Eur. Phys. J. B 23, 221 (2001).

[8] S. Lee, S. H. Yook, and Y. Kim, Phys. Rev. E 74, 046118 (2006).

[9] M. P. H. Stumpt and C. Wiuf, Phys. Rev. E 72, 036118 (2005).

[10] Sang Hoon Lee, Pan-Jun Kim, and Hawoong Jeong, Phys. Rev. E 73, 016102 (2006).

[11] M. E. J. Newman, Soc. Networks 25, 83 (2003).

[12] M. E. J. Newman, Phys. Rev. E. 64, 016132 (2001).

[13] R. Sedgwick, Algorithms (Addison-Wesley, 1988).
[14] K.-I. Goh, B. Kahng, and D. Kim, Phys. Rev. Lett. 87, 278701 (2001).

[15] A. -L. Barabási and R. Albert, Science 286, 509 (1999).

[16] D. Stutzbach and R. Rejaie, Capturing Accurate Snapshots of the Gnutella Networks, In Global Internet Symposium, 127 Mar. (2005); Characterizing the Two Tier Gnutella Topology, In SIGMETRICS, Extended Abstract, June (2005); Characterizing Churn in Peer-toPeer Networks, Tech. Rep. CIS-TR-2005-01, University of Oregon, Eugene, OR, Feb. (2005).

[17] S. H. Yook, F. Radicchi, and H. Meyer-Ortmanns, Phys. Rev. E 72, 045105 (2005).

[18] R. Pastor-Satorras, A. Vázquez, and A. Vespignani, Phys. Rev. Lett. 87, 258701 (2001).

[19] M. E. J. Newman, Phys. Rev. Lett. 89, 208701 (2002).

[20] R. Pastor-Satorras, A. Vázquez, and A. Vespignani, Phys. Rev. E 65, 066130 (2002); Z. E. Ravasz and A. -L. Barabási, Phys. Rev. E 67, 026112 (2003); A. Vázquez, Phys. Rev. E 67, 056104 (2003); J. -S. Lee, K. -I. Goh, B. Kahng, and D. Kim, Eur. Phys. J. B 49, 231 (2006).

[21] S. Lee, S. H. Yook, and Y. Kim, submitted to Phys. Rev. E. 